Exp Brain Res (1989) 74: 618-624

\title{
In vitro dark adaptation and preservation of electrical light responses in the retina from bovine eyes
}

\author{
N. Ryba ${ }^{1}$ and R. Uhl ${ }^{2}$ \\ Max-Planck-Institut für biophysikalische Chemie, Am Faßberg, D-3400 Göttingen, Federal Republic of Germany
}

Summary. A method is described which allows the in vitro dark adaptation of rod photoreceptors from cattle eyes, enucleated under ambient light in the slaughterhouse. Without in vitro dark adaptation these eyes are light adapted and cannot be used for certain delicate biochemical studies and for an electrophysiological characterisation of rod responses. The method is very simple and yields large amounts of dark adapted retinal material, allowing experiments that require bulk amounts of photoreceptor cells. The only source of dark adapted photoreceptors so far have been retinae from dark adapted laboratory animals, which had to be killed and processed under infrared light. Eye cups were opened under red light as soon as possible after their enucleation. Their vitreous humor was removed and their retina thoroughly rinsed with ringer's. Then the eye cup was placed in a moist, light-tight box, where dark adaptation took place. Photoreceptors could thus be kept alive for more than $24 \mathrm{~h}$ without showing signs of deterioration. Humidity and free access of oxygen to the retina were the only prerequisites for their survival. The physiological intactness of the photoreceptors and their degree of dark adaptation was demonstrated by measuring mass receptor potentials (ERGs). A simple device is described which can be used for the electrophysiological characterisation of these eyes.

Key words: In vitro dark adaptation - Bovine retina Electroretinogram (ERG)

\footnotetext{
Present addresses: ${ }^{1}$ Department of Biochemistry, University of Leeds, Leeds LS2 9JT, U.K.

${ }^{2}$ Deptartment of Physiology and Biophysics, Univesity of Washington Seattle, WA 98195, USA

offprint requests to: N. Ryba (address see footnote)
}

\section{Introduction}

While most biochemical studies of the photoreceptor cell make use of material from bovine eyes, electrophysiological experiments rely almost exclusively on amphibian retinas. Bovine photoreceptors are readily obtained in large quantities, but they are small, fragile, and light adapted. The latter is due to the fact that cows are usually killed in the ambient light of a slaughterhouse. No attempt has been made so far to characterise cattle retinae electrophysiologically. Amphibian photoreceptors, on the other hand, are large and sturdy. They are usually obtained from laboratory animals which are sacrificed exclusively for the sake of and immediately prior to a particular experiment. This, and the use of infrared viewing devices, provides for dark adapted photoreceptor cells.

In order to be able to correlate biochemical and electrophysiological data from bovine retinas we have developed a simple and rapid procedure for the in vitro dark adaptation of bovine retinae and we have tested the physiological state of such retinal material using electroretinograms (ERGs) from opened bovine eye cups. The procedure, which is exceedingly simple, yields large amounts of dark adapted photoreceptor rod outer segments, thus facilitating delicate biochemical studies of primary transduction processes which are impossible otherwise (Wagner et al. 1987, 1988a, b, c).

\section{Material and methods}

\section{Preparation and dark adaptation of bovine eye cups}

Bovine eyes (usually between 10 and 20 ) were obtained from a local slaughterhouse. They were - and this is essential - enucleated very briefly (1-3 min) after the death of the animal and stored, at ambient temperature, in a light tight container. Dissection started 


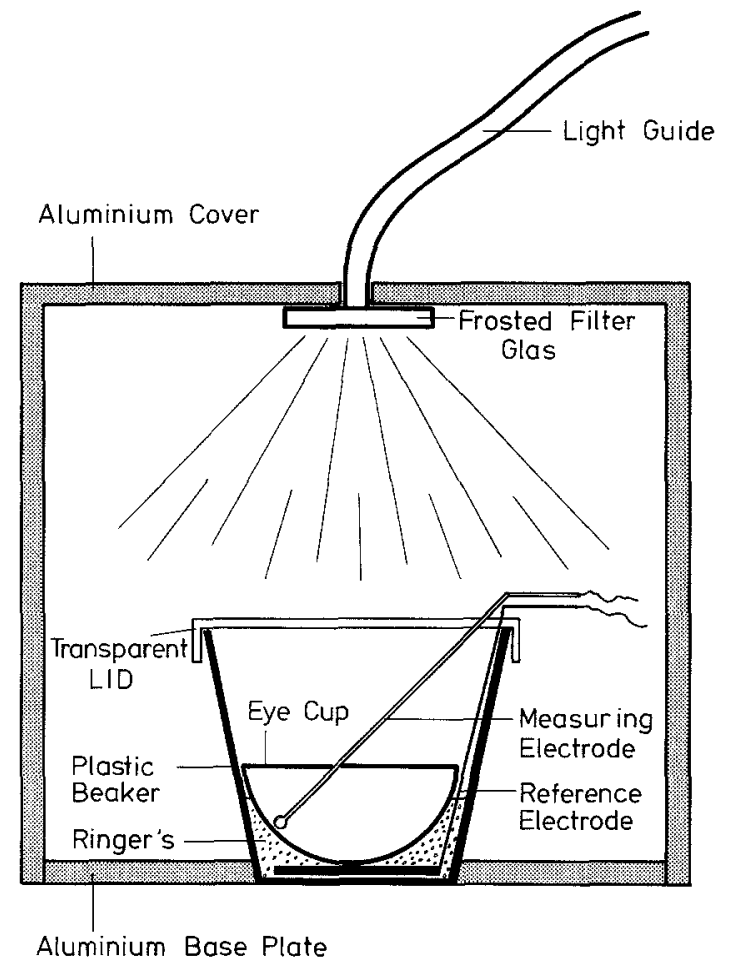

Fig. 1. Schematic drawing of the apparatus for the recording of ERGs from bovine eye cups

no later than 30-40 min after the death of the first animal, and it took place under red light (OSRAM 4563 light bulb). Due to the ability of the eyecups to dark adapt, which will be demonstrated below, infrared viewing devices proved unnecessary.

Each eye was cut in two halves by a circumferential cut along the iris. The posterior part of the eye ball was cautiously agitated until the vitreous humor was released and the retinal surface exposed. It was thoroughly rinsed with a Ringer's solution (containing $10 \%$ bovine fetal serum, $120 \mathrm{mM} \mathrm{NaCl}, 3.5 \mathrm{mM} \mathrm{KCl}$, $10 \mathrm{mM}$ glucose, $10 \mathrm{mM}$ HEPES, pH 7.4, $0.2 \mathrm{mM} \mathrm{CaCl}_{2}, 0.2$ $\mathrm{MgCl}_{2}$, and $0.1 \mathrm{mM} \mathrm{EDTA}$ ) and subsequently placed in a lighttight, humid chamber, were dark adaptation took place within a few minutes. Eyes could be kept there for more than $24 \mathrm{~h}$ without showing signs of deterioration.

\section{Recording of ERGS}

For the recording of electroretinograms (ERGs), an eye cup was rinsed with Ringer's and placed, retina side up, in a plastic beaker, containing $10 \mathrm{ml}$ of Ringer's and a silver/silverchloride ground electrode (Fig. 1). The measuring electrode was a silver wire (1 $\mathrm{mm}$ diameter), the tip of which had been melted to form a smooth, sperical surface of about $2 \mathrm{~mm}$ diameter. It was also chlorided. The electrode fitted through a small hole in the transparent lid of the beaker and was positioned on the retina at an angle of $\mathrm{ca} .45^{\circ}$. It was held in place by friction and its own weight. The whole set was covered by a solid, cylindrical aluminum hood, which provided electrical shielding, kept stray light from reaching the eye and held the light guide for the actinic light in place. All experiments were carried out at room temperature.
Mass potentials from the above setup were amplified in a TEKTRONIX 7A22 amplifier (DC-100 Hz). They were digitised, stored and later inspected in a LSI 11-23 based transient recorder, which has been described elsewhere (Uhl et al. 1984).

Actinic light was brought to the set up by a two-armed light guide, which combined the light from a continuous (adapting) light source (a $50 \mathrm{~W}$ quartz halogen lamp) with that of a xenon test flash. While the latter had a duration of $0.2 \mathrm{~ms}$, the former could be turned on and off in a defined way using a spring loaded mechanical shutter. When the $0.2 \mathrm{~ms}$ test flash was replaced by a short pulse (200 ms) of continuous light, ERGs of similar shape and sensitivity were observed.

Both light sources could be attenuated with neutral density filters and/or spectrally shaped with the help of interference filters. Usually an actinic wavelength of $516 \pm 5 \mathrm{~nm}$ was chosen. Identical ERGs, however, were obtained with an excitation wavelength of $414 \mathrm{~nm}$ or $573 \mathrm{~nm}$, provided the flash energy was raised such that the lower extinction coefficient of rhodopsin at the respective wavelengths was accounted for (data not shown). This clearly indicates that the ERGs displayed in this study reflect responses from rod photoreceptors rather than from cones.

In all experiments a frosted glass surface between the light guide and the retina prevented inhomogeneous illumination.

To determine the bleaching power of the unattenuated light sources, eyecups were exposed to a series of flashes or to defined periods of continuous light, and ROS were isolated from these eye cups immediately afterwards, following a previously published protocol (Uhl et al. 1987). The amount of bleached rhodopsin in these ROS was then determined spectroscopically. The spectral ratio $\mathrm{A}_{280} / \mathrm{A}_{500}$, which is ca. 2.3 in dark adapted ROS (Uhl et al. 1987), increases with light adaptation, and since the absorbance at $500 \mathrm{~nm}$ arises solely from rhodopsin, whereas the absorbance at $280 \mathrm{~nm}$ reflects all proteins of the ROS, $\mathrm{A}_{280} / \mathrm{A}_{500}$ is a convenient measure of the amount of bleached rhodopsin in the ROS. It should be ca. 4.6 , for example, in the case of a $50 \%$ bleached preparation.

\section{Results}

\section{$A$-waves and $b$-waves from dark adapted eye cups}

Typical ERGs, recorded from an eye cup after 30 min of dark adaptation (following the above procedure) are depicted in Fig. 2a and b. Usually the responses consisted mainly of a-waves (Granit's PIII) (Granit 1955). The b-wave component was small and its relative amplitude variable, depending on the preparation and on the position of the electrode on the retina. B-wave threshold was around 1 photoisomerisation per 100 rods (Fig. 2b). A-waves, on the other hand, which directly reflect the photoreceptor response (Sillman et al. 1969), were far more reproducible. Their threshold was slightly higher (1 bleached rhodopsin per 25 rods, Fig. 2a) and saturation occurred not until more than 100 photons were absorbed per rod. Similar values have been reported for rat, toad and skate (Cone 1963; Leibovic et al. 1987; Dowling and Ripps 1972).

When the eye cup was treated with a Ringer's containing $10 \mathrm{mM}$ aspartate, the (already small) 


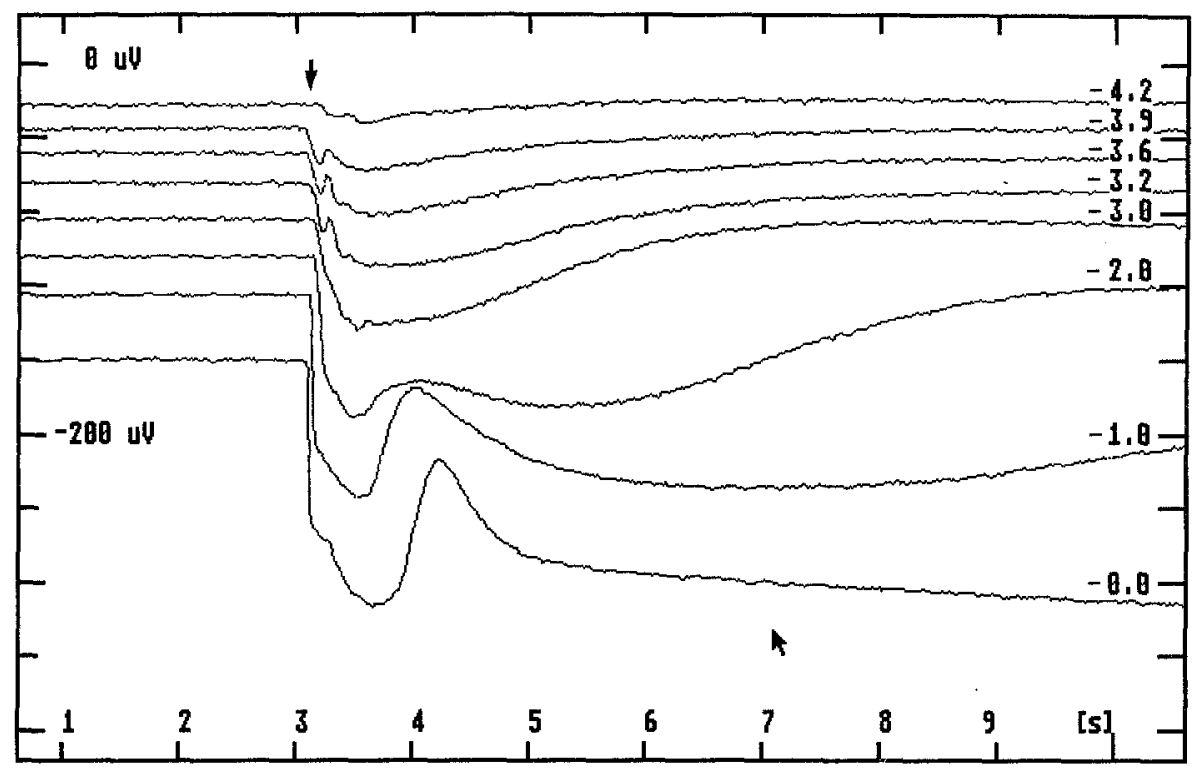

a

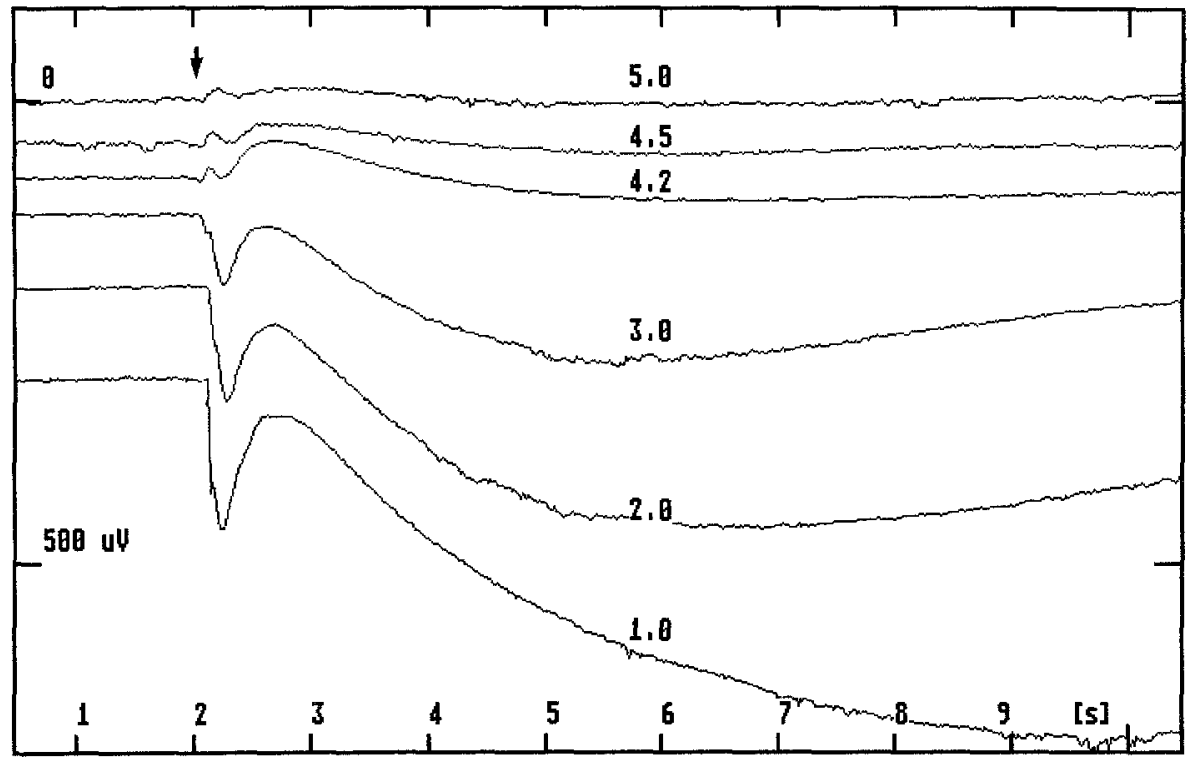

b

contributions of the neural retina to the responses (bwaves) completely vanished. Like in frog and skate (Sillman et al. 1969; Dowling and Ripps 1972), fully receptor dominated responses resulted (Fig. 3a). Similar ERGs were recorded from an $8 \mathrm{~h}$ old eye ball that had not been rinsed with Ringer's, neither prior to its storage, nor prior to the registration of the ERG (Fig. 3b): b-waves were virtually absent. When the same retina was subsequently rinsed with Rin- ger's, however, signs of a b-wave gradually reappeared (Fig. 3c, d). This suggests that during storage in the humid chamber the retina runs out of glucose and that the neural retina is more susceptible to this "starvation" than the photoreceptors themselves. However, since our main interest was the contribution of the photoreceptor cells to the ERG, the awaves, the properties of the small $b$-waves were not explored any further. 2a, b. ERGs from freshly dark small b with more pronounced b-waves. Flashes of increasing energy were applied at a frequency of a $\min ^{-1}$. The numbers denote log attenuation of the maximal flash energy. -5.0 log units correspond to a bleach of 1 rhodopsin/100 rods 

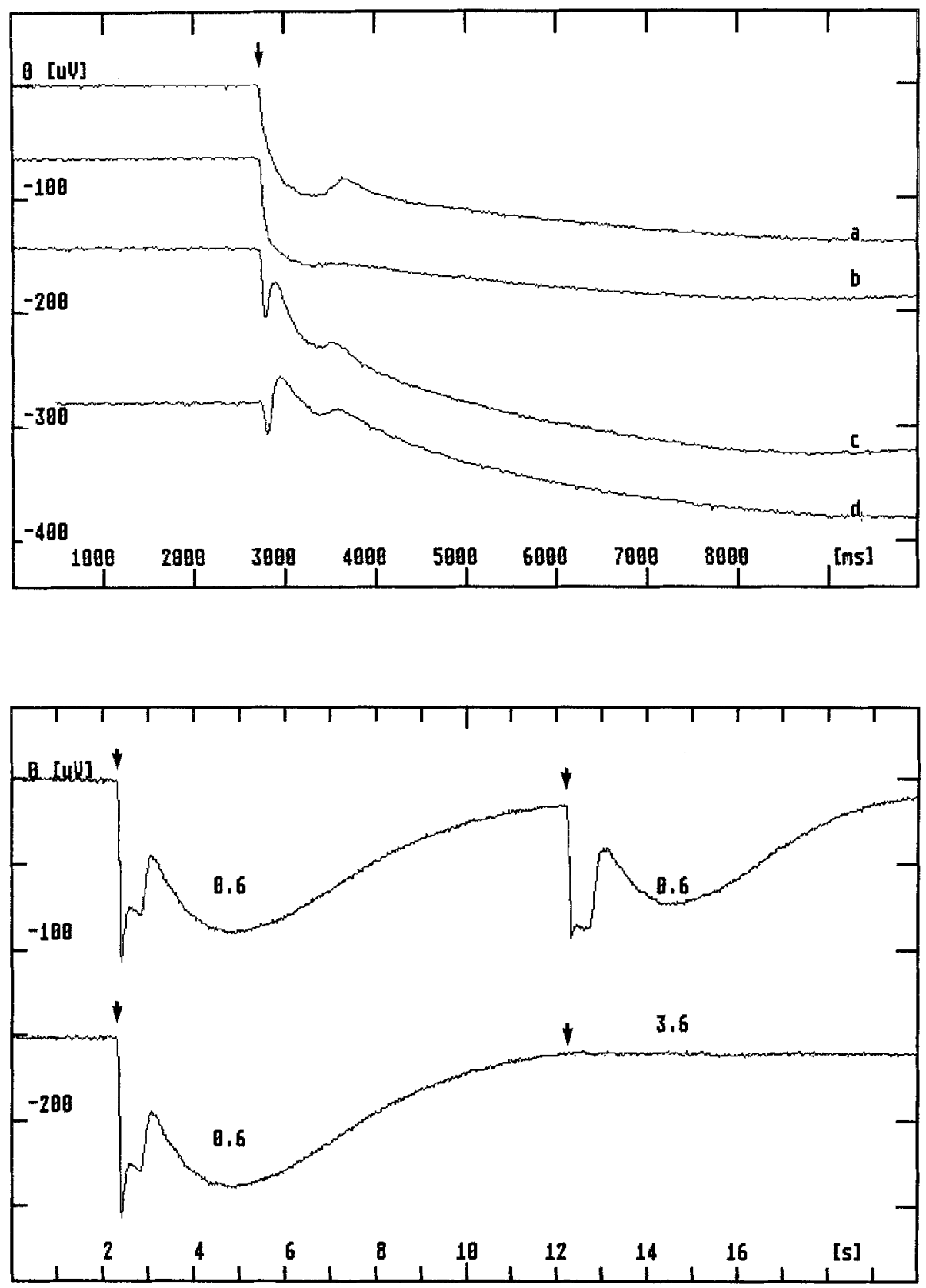

Fig. 3. ERGs (half maximal amplitude) from differently treated eye cups. $a$ Ringer's contained $10 \mathrm{mM}$ aspartate, $b$ the eye cup was never rinsed with Ringer's, $c$ same eye cup as in $\mathbf{b}$, but $1 \mathrm{~min}$ after rinsing it with Ringer's, $d$ same as in $\mathbf{d}$, only 10 min later
Fig. 4. ERGs from a sequence of two light flashes, $10 \mathrm{~s}$ apart. $a$ Two almost saturating flashes (attenuation $-0.6 \log$ units), $b$ one strong flash $(-0.6)$ followed by a weak flash $(-3.6)$, which was strong enough to evoke a half maximal response in the dark adapted eye cup. Lane b was recorded $1 \mathrm{~min}$ after lane a, showing that complete signal recovery can be achieved

\section{Demonstration of light and dark adaptation}

Shortly after a saturating flash a second big blash produced a smaller response (Fig. 4, upper trace). However, within less than a minute the original response size was recovered (lower trace). Weak flashes shortly after a bright flash failed to evoke any response (Fig. 4, lower trace), but after 30-60 s, depending on the energy of the preceding flash, full sensitivity was restored (data not shown). In the presence of a continuous background light (bleaching 10 rhodopsin molecules per rod per second) the response sensitivity was transiently (and reversibly) reduced (Fig. 5), and a flash bleaching $20 \%$ of the total rhodopsin caused a large suppression in sensitivity (Fig. 6), which was only partially reversed within 8 min of dark adaptation.

\section{Stability of the response}

During an experiment lasting $1 \mathrm{~h}$, with some fourty test flashes of varying energy the response to a saturating flash became slightly smaller but stayed 


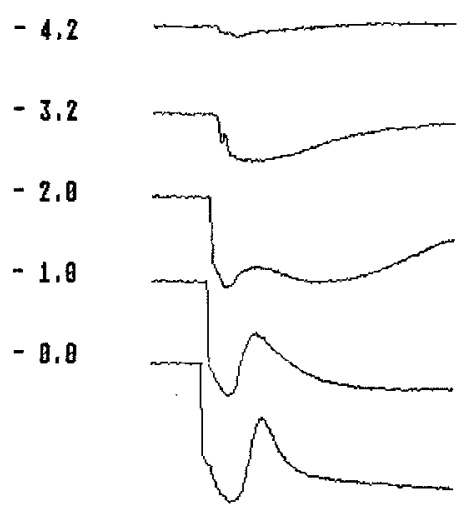

Before

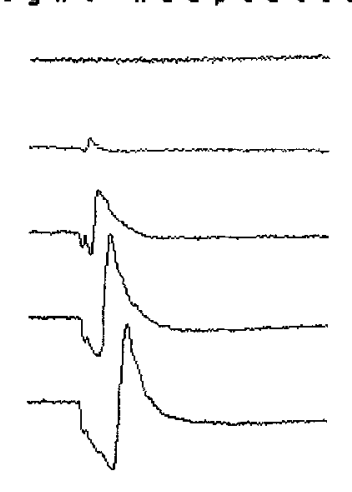

1 in in.

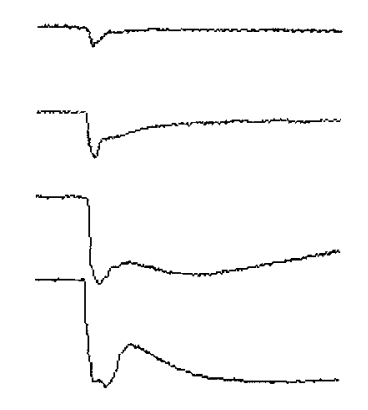

Fig. 5. Adaptation of the ERG to a steady background light. ERGs were measured at different test flash energies before, during and immediately after $30 \mathrm{~min}$ exposure to a dim background light, causing 10 photoisomerisations per second

Ad a ptational Flash

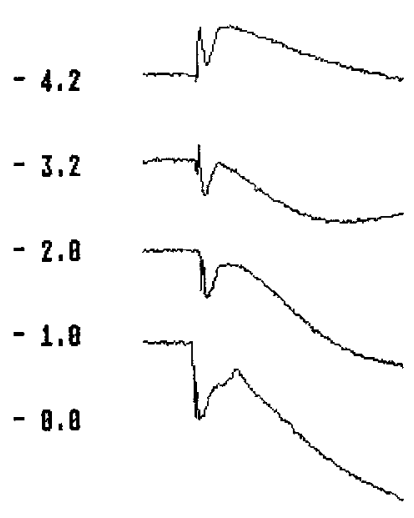

otherwise unchanged (Fig. 7a, b). After $12 \mathrm{~h}$ of dark adaptation in a humid chamber similar ERGs were still obtained (Fig. 7c), whereas eye cups that had been stored for the same amount of time in an intact form, i.e. without exposing their retina to air, were dead (Fig. 7d). This was most likely due to oxygen deprivation, starting as soon as the blood supply was cut off.

\section{Discussion}

When bovine eyes are enucleated in the slaughterhouse they are light adapted to a variable degree. For obvious reasons this is unavoidable. However, given the evidence provided in this communication, there appears to be a way of dark adapting these eyes (or at

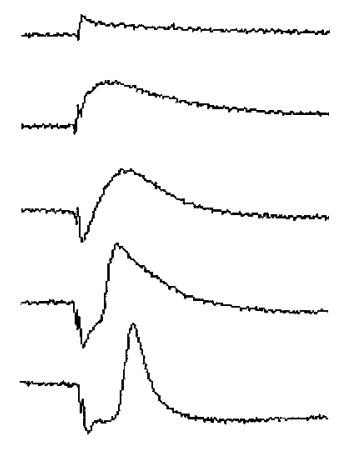

Fig. 6. Adaptation of the ERG after a substantial bleach. ERGs were measured at different test flash energies before, 1 min after and 8 min after a bright flash of green light, bleaching $20 \%$ of the total rhodopsin least their photoreceptors) in vitro, using a very simple and convenient procedure. While the advantages of this procedure for an in vivo - like biochemistry have been critically dealt with elsewhere (Wagner et al. 1987, 1988a, b, c), an attempt has been made in this communication to prove the survival of adaptive properties of the ROS electrophysiologically, using mass receptor potentials (ERGs) from differently treated eye cup preparations.

ERGs from in vitro dark adapted bovine eye cups are rod dominated, as can be seen from their spectral sensitivity. They exhibit large, reproducible a-waves, but small and variable b-waves (Fig. 2), and they closely resemble ERGs from slices of the rat retina. Such slices have been found to produce much smaller b-waves than the living eye or the entire retina 

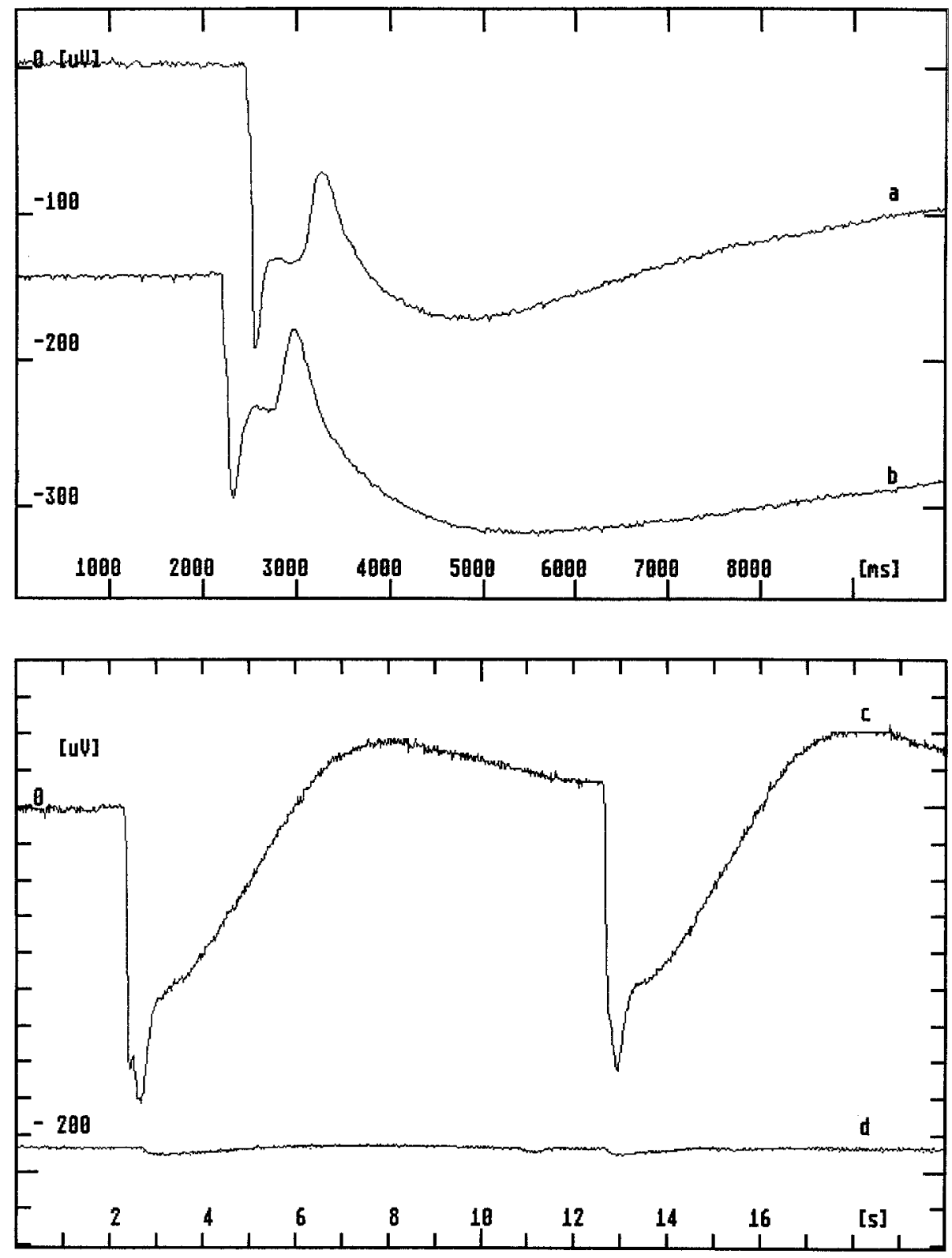

Fig. 7. Stability of the ERG with time. ERGs evoked by a saturating flash, recorded: $a$ at the begin, $b$ at the end of a sequence of measurements lasting $1 \mathrm{~h}, c$ from a $12 \mathrm{~h}$ old retina, dark adapted as described, $d$ from a 12 h old retina, "dark adapted" in the undissected eye
(Hagins et al. 1970). Since the middle and the inner layer of the retina, which are responsible for the bwave, were not of interest in the context of this study, the reasons for the lack of reproducibility of b-waves were not further explored. Instead it was focused on the a-wave (or PIII), which has been shown to reflect photoreceptor activity directly (Sillman et al. 1969).

The observed response threshold was low, similar to the values found in other dark adapted retinae. Bwaves, for instance, - provided they were measurable - had a threshold value of 1 bleached rhodopsin per 100 rods, and a-waves could be detected when 1 rhodopsin was bleached per 25 rods. Very similar values $\left(1 R^{*} / 200\right.$ rods in the case of b-waves and $I R^{*} / 5$ rods for a-waves) have been reported for the dark adapted rat retina (Cone 1963). The low values found in our study clearly point to fully dark adapted photoreceptor system.

Both light and dark adaptations have been directly visualized in Figs. 4, 5, and 6. There it was demonstrated that both preilumination and background illumination change the sensitivity of the photoreceptors and that the reversibility of this effect depends on the level of bleaching. This is further support for our claim that the photoreceptors in our eye cup preparation are "alive".

Finally it should be mentioned that yet another feature of the "living photoreceptor" is preserved in 
our eye cup preparation, a light migration of SAntigen, the $48 \mathrm{k}$-protein of the photoreceptor cell from the inner to the outer segment. This, and its influence on adaptation processes (revealed by ERGs) will be dealt within a separate communication (N. Ryba and R. Uhl, in preparation).

Acknowledgement. The authors wish to express their gratitude to Prof. W. Sickel for many hints and helpful suggestions.

\section{References}

Cone RA (1963) Quantum relations of the rat electroretinogram. J Gen Physiol 46: 1267-1286

Dowling JE, Ripps H (1972) Adaptation in skate photoreceptors. J Gen Physiol 60: 698-719

Granit R (1955) Receptors and sensory perception. Yale Univer sity Press, New Haven

Hagins WA, Penn RD, Yoshikami S (1970) Dark current and photocurrent in retinal rods. Biophys J 10: 380-412

Leibovic KN, Dowling UJE, Kim YY (1987) Background and bleaching equivalence in steady state adaptation of vertebrate rods. J Neurosci 7: 1056-1063
Sillman RJ, Ito H, Tomita T (1969) Studies on the mass receptor potential of isolated frog retina. I. General properties of the response. Vision Res 9: 1435-1442

Uhl R, Meyer B, Desel H (1984) A polychromatic flash photolysis apparatus (PFPA). J Biochem Biophys Methods 10: 35-48

Uhl R, Desel H, Ryba N, Wagner R (1987) Simple and rapid procedure for the isolation of intact bovine rod outer segments (ROS). J Biochem Biophys Methods 14: 127-138

Wagner R, Ryba N, Uhl R (1987) The amplified P-Signal, an extremely photosensitive light scattering signal from rod outer segments, which is not affected by preactivation of phosphodiesterase with $\mathrm{G}_{\alpha}$-GTP- $\gamma-\mathrm{S}$. FEBS Lett 221: 253-259

Wagner R, Ryba N, Uhl R (1988) Subsecond turnover of transducin GTPase in bovine rod outer segments. FEBS Lett 234: 44-48

Wagner R, Ryba N, Uhl R (1988) Rapid transducin deactivation in intact stacks of bovine rod outer segment disks as studied by light scattering techniques. FEBS Lett 235: 103-108

Wagner R, Ryba N, Uhl R (1988) Calcium regulates the rate of rhodopsin disactivation and the primary amplification step in visual transduction. FEBS Lett (in press)

Received May 31, 1988 / Accepted October 13, 1988 